\title{
SPECTRAL DUALITY AND DISTRIBUTION OF EXPONENTS FOR TRANSFER MATRICES OF BLOCK TRIDIAGONAL HAMILTONIANS
}

\author{
(revised, february 2003)
}

\author{
Luca Molinari \\ Dipartimento di Fisica dell'Università degli Studi di Milano \\ and I.N.F.N., sezione teorica di Milano. Via Celoria 16, I-20133 Milano \\ luca.molinari@mi.infn.it
}

\begin{abstract}
:
I consider a general block-tridiagonal matrix and the corresponding transfer matrix. By allowing for a complex Bloch parameter in the boundary conditions, the two matrices are related by a spectral duality.

As a consequence, I derive some analytic properties of the exponents of the transfer matrix in terms of the eigenvalues of the (non-Hermitian) block matrix. Some of them are the single-matrix analogue of results holding for Lyapunov exponents of an ensemble of block matrices, that occur in models of transport. The counting function of exponents is related to winding numbers of eigenvalues. I discuss some implications of duality on the distribution (real bands and complex arcs) and the dynamics of eigenvalues.
\end{abstract}

P.A.C.S.: 02.10.Yn (matrix theory), 72.15.Rn (localization effects), 72.20.Ee (mobility edges, hopping transport)

\section{Introduction}

A tridiagonal Hermitian matrix whose entries are square matrices of size $M$ is a block tridiagonal matrix. By denoting $H_{i}=H_{i}^{\dagger}$ the diagonal blocks and by $L_{i}$ the blocks in the next upper diagonal, the eigenvalue equation written in the block components of an eigenvector $\underline{u}$ is:

$$
H_{i} \underline{u}_{i}+L_{i} \underline{u}_{i+1}+L_{i-1}^{\dagger} \underline{u}_{i-1}=E \underline{u}_{i}
$$

We may view $H_{i}$ as Hamiltonian matrices of a chain of subsystems, each with $M$ internal states, sequentially coupled by matrices $L_{i}$. We shall make the only requirement $\operatorname{det} L_{i} \neq 0$.

Band matrices are in this class, with non-diagonal blocks being triangular. Random band matrices are studied for quantum chaos and transport, mainly by numerical means [1] or by a mapping on a non-linear supersymmetric sigma model [2]. The block structure is typical of tight binding models, as Anderson's model for the transport of a particle in a lattice with impurities [3]. Here, the matrices $H_{i}$ are the Hamiltonians of isolated slices transverse to some direction and the matrices $L_{i}$ contain the hopping amplitudes between lattice sites of neighboring slices. Tridiagonal arrays of random matrices were studied in the context of 
multichannel scattering in mesoscopic or nuclear physics [4] or as multi-matrix models in the large size limit [5]. For recent reviews of applications of random matrices in physics, see $[6,7]$.

The second-order recursive character of (1) makes it useful to introduce a transfer matrix $T_{i}(E)$, of size $2 M \times 2 M$

$$
\left(\begin{array}{c}
\underline{u}_{i+1} \\
\underline{u}_{i}
\end{array}\right)=\left(\begin{array}{cc}
L_{i}^{-1}\left(E-H_{i}\right) & -L_{i}^{-1} L_{i-1}^{\dagger} \\
I & 0
\end{array}\right)\left(\begin{array}{c}
\underline{u}_{i} \\
\underline{u}_{i-1}
\end{array}\right)
$$

The block components of the eigenvector are reconstructed by applying a product of transfer matrices to an initial block pair. A length $N$ of the chain corresponds to the transfer matrix $T(E)=T_{N}(E) \ldots T_{1}(E)$ :

$$
\left(\begin{array}{c}
\underline{u}_{N+1} \\
\underline{u}_{N}
\end{array}\right)=T(E)\left(\begin{array}{c}
\underline{u}_{1} \\
\underline{u}_{0}
\end{array}\right)
$$

The transfer matrix is the main tool for investigating boundary properties of the Hamiltonian's eigenvectors, or the transmission matrix of the chain coupled to leads. I developed a theory for transfer matrices of general block-tridiagonal Hamiltonians in $[8,9]$. Recently the formalism was applied to transport in nanotubes and molecules and generalized to allow for non-invertible off-diagonal blocks $[10,11,12,13]$.

In this paper I describe some interesting consequences of a nice algebraic identity involving the characteristic polynomials of the two matrices [8]. Though this spectral duality holds in general, I here restrict to Hermitian block tridiagonal matrices because of their relevance in physics.

For a chain of length $N$ we must provide boundary conditions. With periodic boundary conditions we require $L_{0}=L_{N}$ for Hermiticity and $\underline{u}_{0}=\underline{u}_{N}, \underline{u}_{N+1}=$ $\underline{u}_{1}$. However, it turns to be very convenient to allow for a complex Bloch parameter:

$$
\underline{u}_{0}=\frac{1}{z} \underline{u}_{N}, \quad \underline{u}_{N+1}=z \underline{u}_{1}
$$

Therefore the Hamiltonian matrix is block-tridiagonal with corners:

$$
\mathcal{H}(z)=\left(\begin{array}{ccccccc}
H_{1} & L_{1} & & & & & \frac{1}{z} L_{N}^{\dagger} \\
L_{1}^{\dagger} & H_{2} & L_{2} & & & & \\
& L_{2}^{\dagger} & \ldots & \ldots & & & \\
& & & & \ldots & \ldots & L_{N-1} \\
z L_{N} & & & & & L_{N-1}^{\dagger} & H_{N}
\end{array}\right)
$$

It is Hermitian only for $|z|=1$. Boundary conditions with $z=e^{i \varphi}$ arise when decomposing the eigen-problem (1) for an infinite periodic chain of period $N$ in the eigen-spaces of the $N$-block translation operator, as well as in the topology of a $N$-site ring with a magnetic flux through it. In general it is:

$$
\mathcal{H}(z)^{\dagger}=\mathcal{H}\left(1 / z^{*}\right)
$$


Non Hermitian tridiagonal matrices $(M=1)$, with $z=e^{N g}$ and $g \geq 0$, were introduced by Hatano and Nelson [14] in a study on vortex depinning in superconductors which promoted a burst of research, see for example $[15,16,17,18]$. Spectral properties were analyzed in greater detail by Goldsheid and Khoruzhenko [19] who proved that, for $g>g_{c r}$, eigenvalues corresponding to extended states begin to migrate in the complex plane and distribute along the level curve of the single Lyapunov exponent of the model: $\gamma(E)=g$. Two wings of real eigenvalues correspond to exponentially localized eigenvectors, that are insensitive to the boundary.

These features also appear in the more difficult case of block matrices. For $|z|$ sufficiently greater or smaller than unity, the block matrix develops complex eigenvalues which are seen numerically to distribute along lines. The eigenvalues of an eptadiagonal matrix $(\mathrm{M}=3)$ with diagonal disorder and unit hopping amplitudes, are shown in Fig.2.

It is intuitive that there is a connection between boundary properties of eigenvectors, which are described by the transfer matrix, and the response of energy eigenvalues to variations of boundary conditions: namely, the Landauer and Thouless approaches to transport. This intuition has a formulation in the spectral duality.

After a short review of duality (section 2), I derive several analytic properties of the exponents of a single transfer matrix (section 3). In particular, I evaluate the counting function of exponents as the winding number of trajectories of eigenvalues of the source block matrix. In section 4 , I use duality to describe qualitatively the distribution and dynamics of eigenvalues of Hermitian and non-Hermitian block-tridiagonal matrices.

\section{Spectral Duality}

In this section I review some basic properties of a transfer matrix $T(E)$ of a chain of length $N$, with $L_{0}=L_{N}[8,9]$.

$T(E)$ is a matrix polynomial in $E$ of degree $N$, with a non-zero determinant independent of $E$ and of diagonal blocks $H_{i}$ :

$$
\operatorname{det} T(E)=\prod_{i=1}^{N} \frac{\operatorname{det} L_{i}^{\dagger}}{\operatorname{det} L_{i}}
$$

The block structure of the Hamiltonian and the corresponding factorization of the transfer matrix, makes a typical property of transfer matrices apparent:

Proposition 1: the symplectic property.

$$
T\left(E^{*}\right)^{\dagger} \Sigma_{N} T(E)=\Sigma_{N}, \quad \Sigma_{N}=\left(\begin{array}{cc}
0 & -L_{N}^{\dagger} \\
L_{N} & 0
\end{array}\right)
$$


Proof: it is a consequence of $L_{0}=L_{N}$ and of the factorization of $T(E)$ into a product of matrices $T_{k}(E)$, whose inverse is: $T_{k}(E)^{-1}=\Sigma_{k-1}^{-1} T_{k}\left(E^{*}\right)^{\dagger} \Sigma_{k}$.

Corollary: if $z$ is an eigenvalue of $T(E)$, then $1 / z^{*}$ is an eigenvalue of $T\left(E^{*}\right)$. For real $E$, both $z$ and $1 / z^{*}$ are in the spectrum of $T(E)$. If $T\left(E^{*}\right)=T(E)^{*}$, then both $z$ and $1 / z$ are in the spectrum of $T(E)$.

These statements summarize in the useful identity:

$$
\operatorname{det}[T(E)-z]=z^{2 M} \operatorname{det} T(0) \operatorname{det}\left[T\left(E^{*}\right)-1 / z^{*}\right]^{*}
$$

Let $\underline{u}$, with blocks $\underline{u}_{1}, \ldots, \underline{u}_{N}$, be an eigenvector of $\mathcal{H}(z)$ with eigenvalue $E$. Then, by eq. (3) and after imposing the boundary conditions (4):

$$
\left(\begin{array}{l}
z \underline{u}_{1} \\
\underline{u}_{N}
\end{array}\right)=T(E)\left(\begin{array}{c}
\underline{u}_{1} \\
1 / z \underline{u}_{N}
\end{array}\right)
$$

which means that $z$ is an eigenvalue of $T(E)$ with an eigenvector of blocks $z \underline{u}_{1}$ and $\underline{u}_{N}$. However the converse is true: given an eigenvector of $\mathrm{T}(\mathrm{E})$ with eigenvalue $z$ one reconstructs via products of matrices $T_{k}(E)$ the whole eigenvector of $\mathcal{H}(z)$ with eigenvalue $E$. Therefore $\operatorname{det}[E-\mathcal{H}(z)]=0$ if and only if $\operatorname{det}[T(E)-z]=0$; this duality among eigenvalues is made precise as an identity among characteristic polynomials [8]:

Proposition 2: the spectral duality.

$$
\operatorname{det}[E-\mathcal{H}(z)]=(-z)^{-M} \operatorname{det}\left[L_{1} L_{2} \ldots L_{N}\right] \operatorname{det}[T(E)-z]
$$

Proof: we must show that $\operatorname{det}[T(E)-z]$ is a polynomial in $E$ of degree $N M$ with leading coefficient $(-z)^{M} \operatorname{det}\left[L_{1} \ldots L_{N}\right]^{-1}$. To this end, we first consider the leading terms in $E$ of both sides of eq.(9): the equality implies that the leading term of $\operatorname{det}[T(E)-z]$ is proportional to $z^{M}$. Next we derive the following leading block stucture of $T(E)$ :

$$
T(E) \approx\left(\begin{array}{cc}
E^{N} Q & -E^{N-1} Q L_{N}^{\dagger} \\
E^{N-1} L_{N} Q & -E^{N-2} L_{N} Q L_{N}^{\dagger}
\end{array}\right), \quad Q=\left(L_{1} \ldots L_{N}\right)^{-1}
$$

The leading term in $E$ of $\operatorname{det}[T(E)-z I]$ with the constraint of being proportional to $z^{M}$, is provided by the diagonal factors $\operatorname{det}\left(E^{N} Q\right)$ and $\operatorname{det}(-z I)$.

Corollary: If $\operatorname{Im} E \neq 0$ then $T(E)$ has no eigenvalues on the unit circle.

Proof: for $|z|=1$ the matrix $\mathcal{H}(z)$ is Hermitian, and for $\operatorname{Im} E \neq 0$ it is always $\operatorname{det}[E-\mathcal{H}(z)] \neq 0$. By duality, this implies $\operatorname{det}[T(E)-z] \neq 0$.

Notes: in [9] I provided a representation of $T(E)-z$ in terms of the corner blocks of the resolvent of $\mathcal{H}(z)$. I also state the spectral duality for the matrix $T^{\dagger} T$. 
In the tridiagonal case $(M=1)$, blocks are numbers. If $\lambda=L_{1} \ldots L_{N}$ the spectral duality simplifies to the known expression

$$
\operatorname{det}[E-\mathcal{H}(z)]=\lambda \operatorname{tr} T(E)-\lambda z-\lambda^{*} \frac{1}{z}
$$

\section{The spectrum of exponents}

Let us fix $E$ real or complex and denote as $z_{a}(E)=e^{\xi_{a}+i \varphi_{a}}, a=1 \ldots 2 M$, the eigenvalues of $T(E)$. The real numbers $\xi_{a}(E)$ are the exponents of the transfer matrix. For real $E$ the symplectic property (8) assures that exponents come in pairs $\pm \xi_{a}(E)$.

The property $|\operatorname{det} T(E)|=1$, see (7), implies:

$$
\sum_{a=1}^{2 M} \xi_{a}(E)=0
$$

When considering an ensemble of random Hamiltonian matrices with tridiagonal block structure, one is often interested in the corresponding ensemble of transfer matrices. Being a product of $N$ random matrices, a transfer matrix develops exponents $\xi_{a}(E)$ that asymptotically grow linearly in the length $N$ [20], with a coefficient known as the Lyapunov exponent:

$$
\gamma_{a}(E)=\lim _{N \rightarrow \infty} \frac{1}{N}\left\langle\xi_{a}(E)\right\rangle
$$

For tridiagonal random matrices there is just one pair of opposite Lyapunov exponents, that can be evaluated with the Herbert-Jones-Thouless formula [21], with the knowledge of the average density of eigenvalues:

$$
\gamma(E)=\text { const }+\int d E^{\prime} \rho\left(E^{\prime}\right) \log \left|E-E^{\prime}\right|
$$

The extension to a complex value $E$ is discussed in [22].

For the Anderson model [23] or Band Random Matrices [24] there are several numerical studies of Lyapunov spectra. In these cases of great physical interest, transfer matrices are derived from the Hamiltonians and the analytic approach is difficult.

It is interesting that an analytic formula relating the distribution of the exponents to the spectrum of the Hamiltonian is possible. Note that the following statements are true for a single and general block-tridiagonal Hamiltonian. We shall deduce several results from

\section{Proposition 3:}

$$
\int_{0}^{2 \pi} \frac{d \varphi}{2 \pi} \log \left|\operatorname{det}\left[E-\mathcal{H}\left(e^{\xi+i \varphi}\right)\right]\right|=\sum_{i=1}^{N} \log \left|\operatorname{det} L_{i}\right|+\frac{1}{2} \sum_{a=1}^{2 M}\left|\xi-\xi_{a}(E)\right|
$$


Proof: The duality relation (11) gives:

$$
\begin{array}{r}
\log \left|\operatorname{det}\left[E-\mathcal{H}\left(e^{\xi+i \varphi}\right)\right]\right|-\sum_{i} \log \left|\operatorname{det} L_{i}\right|= \\
=-M \xi+\frac{1}{2} \sum_{a=1}^{2 M} \log \left|e^{\xi_{a}+i \varphi_{a}}-e^{\xi+i \varphi}\right|^{2} \\
=\frac{1}{2} \sum_{a=1}^{2 M}\left\{\xi_{a}+\log \left[2 \cosh \left(\xi_{a}-\xi\right)-2 \cos \left(\varphi_{a}-\varphi\right)\right]\right\} \\
=\frac{1}{2} \sum_{a=1}^{2 M}\left|\xi_{a}-\xi\right|-\sum_{\ell=1}^{\infty} \sum_{a=1}^{2 M} \frac{\cos \ell\left(\varphi_{a}-\varphi\right)}{\ell} e^{-\ell\left|\xi_{a}-\xi\right|}
\end{array}
$$

We used eq.(13) and the formula (see eq. 1.448.2 in [25]):

$$
\log [2 \cosh x-2 \cos y]=|x|-2 \sum_{\ell=1}^{\infty} \frac{\cos \ell y}{\ell} e^{-\ell|x|}
$$

Eq. (16) is the Fourier expansion of $\log \left|\operatorname{det}\left[E-\mathcal{H}\left(e^{\xi+i \varphi}\right)\right]\right|$, which is a periodic function of $\varphi$. The constant mode is just the proposition. •

In the special case $\xi=0$, the matrix $\mathcal{H}\left(e^{i \varphi}\right)$ is Hermitian, and (15) yields a formula for the sum of positive exponents ( $E$ can be complex):

\section{Proposition 4:}

$$
\sum_{\xi_{a}>0} \xi_{a}(E)=-\sum_{i} \log \left|\operatorname{det} L_{i}\right|+\int_{0}^{2 \pi} \frac{d \varphi}{2 \pi} \log \left|\operatorname{det}\left(E-\mathcal{H}\left(e^{i \varphi}\right)\right)\right|
$$

By taking the derivative in $\xi$ of (15) we obtain the spectral counting function, which counts the exponents less than $\xi$, for any complex value $E$;

$$
\begin{gathered}
\mathcal{N}\left(\xi_{a}(E) \leq \xi\right)=\sum_{a=1}^{2 M} \theta\left(\xi-\xi_{a}(E)\right)= \\
M+\frac{d}{d \xi} \int_{0}^{2 \pi} \frac{d \varphi}{2 \pi} \log \left|\operatorname{det}\left[E-\mathcal{H}\left(e^{\xi+i \varphi}\right)\right]\right|
\end{gathered}
$$

We now write $|\operatorname{det}[E-\mathcal{H}(z)]|$ in terms of the eigenvalues $E_{n}(z)$ and their complex conjugate, which equal $E_{n}\left(1 / z^{*}\right)$, and evaluate the derivative:

$$
\begin{array}{r}
\frac{d}{d \xi} \log \left|\operatorname{det}\left[E-\mathcal{H}\left(e^{\xi+i \varphi}\right)\right]\right|= \\
=-\frac{1}{2} \sum_{n}\left(\frac{1}{E-E_{n}(z)} \frac{\partial E_{n}(z)}{\partial \xi}+\frac{1}{E^{*}-E_{n}(z)^{*}} \frac{\partial E_{n}(z)^{*}}{\partial \xi}\right) \\
=-\frac{1}{2 i} \sum_{n}\left(\frac{1}{E-E_{n}(z)} \frac{\partial E_{n}(z)}{\partial \varphi}-\frac{1}{E^{*}-E_{n}(z)^{*}} \frac{\partial E_{n}(z)^{*}}{\partial \varphi}\right)
\end{array}
$$


Let us denote with $N_{+}(E)$ and $N_{-}(E)$ the numbers of positive and negative exponents of $T(E)$. We have:

Proposition 5: $N_{+}(E)=N_{-}(E)$

Proof: for real $E$ we know that the exponents come in pairs $\pm \xi_{a}(E)$, because of proposition 1. Let us consider the case $\operatorname{Im} E \neq 0$.

As a consequence of duality we derived that no eigenvalue of $T(E)$ is on the unit circle, thus all exponents are non-zero: $N_{-}+N_{+}=2 M$. Therefore, if we set $\xi=0$ in (19), the left term is $N_{-}$. We now show that $N_{-}=M$ or, equivalently, that the integral in (19) vanishes for $\xi=0$. In the expression (20) the eigenvalues $E_{n}\left(e^{i \varphi}\right)$ are real periodic functions of $\varphi$ in $[0,2 \pi]$, then

$$
N_{-}(E)-M=\sum_{n=1}^{N M} \int_{0}^{2 \pi} \frac{d \varphi}{2 \pi} \frac{d E_{n}}{d \varphi} \frac{\operatorname{Im} E}{\left(\operatorname{Re} E-E_{n}\right)^{2}+(\operatorname{Im} E)^{2}}=0
$$

As a function of $\varphi$ ( $\xi$ is fixed) an eigenvalue $E_{n}\left(e^{\xi+i \varphi}\right)$ makes a loop $\gamma_{n}$ in the complex $E$ plane. The loop $\gamma_{n}^{*}$ of $E_{n}^{*}$ is specular with respect respect of the real axis. Integration in $\varphi$ of (20) yields Cauchy integrals

$$
\mathcal{N}\left(\xi_{a}(E)<\xi\right)=M+\frac{1}{2} \sum_{n}\left(\int_{\gamma_{n}} \frac{d E^{\prime}}{2 \pi i} \frac{1}{E^{\prime}-E}-\int_{\gamma_{n}^{*}} \frac{d E^{\prime}}{2 \pi i} \frac{1}{E^{\prime}-E^{*}}\right)
$$

The first integral is the winding number of the (oriented) trajectory $\gamma_{n}$ of $E_{n}\left(e^{\xi+i \varphi}\right)$ around the value $E$. The second integral is the winding number of $\gamma_{n}^{*}$ around $E^{*}$ and has opposite sign because of opposite orientation.

We then obtain a nice geometric result:

Proposition 6: $\mathcal{N}\left(\xi_{a}(E)<\xi\right)=M+\mathcal{W}(E)$

The number of exponents of $T(E)$ less than $\xi$ equals $M$ plus the total winding number of loops of eigenvalues $E_{n}\left(e^{\xi+i \varphi}\right),-\pi<\varphi<\pi$, that encircle $E$

As I said, these formulae hold for a single general block tridiagonal Hamiltonian matrix and its transfer matrix. For a statistical ensemble of Hamiltonians one performs ensemble averages in place of a phase average, and deals with Lyapunov exponents. Souillard [20] obtained the following formula for the positive Lyapunov exponents, which is the statistical analogue of (17):

$$
\frac{1}{M} \sum_{a} \gamma_{a}(E)=\text { const. }+\int d E^{\prime} \rho\left(E^{\prime}\right) \log \left|E-E^{\prime}\right|
$$

$\rho(E)$ is the ensemble averaged spectral density of the Hermitian Hamiltonians. I am not aware of any statistical analogue of (19).

\section{Bands, arcs and energy level motion.}


Spectral duality provides information on the positions of the eigenvalues of $\mathcal{H}(z)$ and their motion under variations of the boundary parameter $z$.

It is useful to introduce the notion of discriminant. If $z_{a}(E)$ is an eigenvalue of $T(E)$, the discriminant is the eigenvalue of $T(E)+T(E)^{-1}$ with same eigenvector:

$$
\Delta_{a}(E)=z_{a}(E)+\frac{1}{z_{a}(E)}=2 \cosh \xi_{a} \cos \varphi_{a}+2 i \sinh \xi_{a} \sin \varphi_{a}
$$

Since the symplectic property implies that $1 / z_{a}^{*}$ is an eigenvalue of $T\left(E^{*}\right)$, it is, in general:

$$
\Delta_{a}\left(E^{*}\right)=\Delta_{a}(E)^{*}
$$

Let us begin with the simpler case where $H_{i}, L_{i}$ are real matrices. Then $T\left(E^{*}\right)=T(E)^{*}$ and the eigenvalues of the transfer matrix come in pairs $z_{a}$ and $1 / z_{a}, a=1 \ldots M$. Moreover, if $E$ is real the characteristic polynomial of $T(E)$ has real coefficients and roots come also in pairs $z_{a}, z_{a}^{*}$.

The spectral duality reads:

$$
\prod_{a=1}^{M}\left[\left(z_{a}+\frac{1}{z_{a}}\right)-\left(z+\frac{1}{z}\right)\right]=\operatorname{det}\left[L_{1} \ldots L_{n}\right]^{-1} \operatorname{det}[E-\mathcal{H}(z)]
$$

Therefore the $M$ equations

$$
\Delta_{a}(E)=z+\frac{1}{z}
$$

provide the $N M$ eigenvalues of $\mathcal{H}(z)$, which are naturally classified in subsets with label $a$. We consider two cases: $|z|=1$ and $z$ real.

When $z=e^{i \varphi}$ all eigenvalues of $\mathcal{H}\left(e^{i \varphi}\right)$ are real periodic functions of $-\pi \leq$ $\varphi<\pi$. Each equation $\Delta_{a}(E)=2 \cos \varphi$ provides a number $n_{a} \geq 0$ of real solutions, and $n_{1}+\ldots+n_{M}=N M$. This means that $y=\Delta_{a}(E)$, as a function of the real variable $E$, crosses $n_{a}$ times the strip $-2 \leq y \leq 2$ parallel to the $E$ axis. No extrema are allowed in the strip, for all branches must cut $n_{a}$ times the lines $y=2 \cos \varphi$, to ensure that $\mathcal{H}\left(e^{i \varphi}\right)$ has $N M$ real eigenvalues for all $\varphi$. All branches of the functions $\Delta_{a}(E)$ cross their bands in the eigenvalues of $\mathcal{H}(i)$. See Fig. 1.

By changing $\varphi$ the eigenvalues span bands in the real axis

$$
E=E_{a, j}(\varphi) \quad a=1 \ldots M, j=1 \ldots n_{a}
$$

with extrema given by the eigenvalues of the periodic $(\varphi=0)$ and antiperiodic $(\varphi=\pi)$ Hamiltonians. The velociy of level motion is

$$
\frac{\partial E}{\partial \varphi}=-\frac{2 \sin \varphi}{\Delta_{a}^{\prime}(E)}
$$


In the turning points the velocity vanishes. The second derivative is known as the curvature $[1,26,27]$. This dynamics is invariant under the "time-reversal" operation $\varphi \rightarrow-\varphi$, corresponding to the transposition of the Hamiltonian matrix.

Bands with same label $a$ may at most share an extremum, while bands related to different $a$ may overlap.

When a branch of $\Delta_{a}$ and a branch of $\Delta_{a^{\prime}}$ cross inside the strip in a point $(E, 2 \cos \varphi)$, there is a crossing of two eigenvalues of $\mathcal{H}\left(e^{i \varphi}\right)$ (and a collision of two pairs of eigenvalues of $T(E)$ in the unit circle). This is a highly non-generic occurrence for one-parameter Hermitian matrices.

Energy bands of Hermitian periodic tridiagonal matrices $(M=1)$ were studied in $[28]$

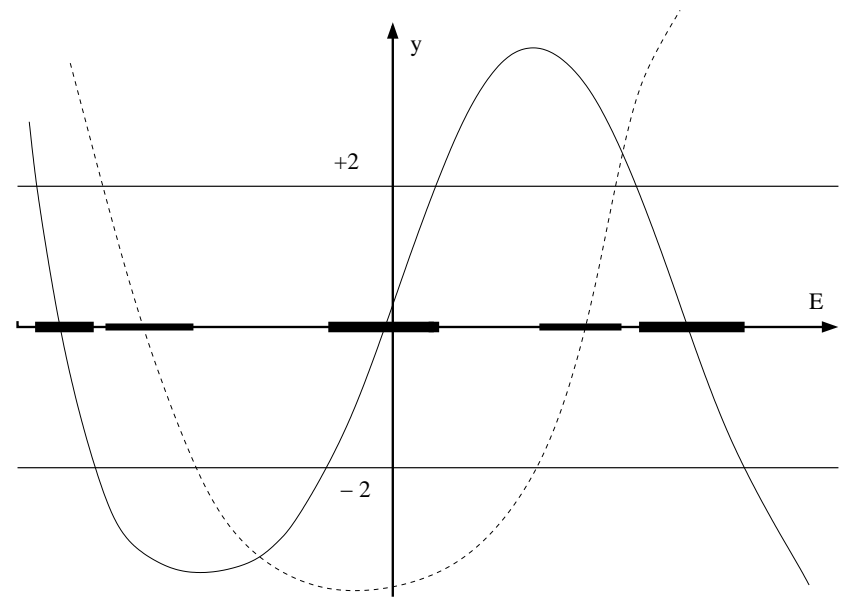

Fig.1 Energy bands of a pentadiagonal matrix $(M=2)$, solution to $\Delta_{a}(E)=$ $2 \cos \varphi, a=1,2,-\pi \leq \varphi<\pi$.

When $z=e^{N g}$, with $g \geq 0$, the matrix $\mathcal{H}(z)$ is real. The equations $\Delta_{a}(E)=$ $2 \cosh (N g)$ provide all $N M$ eigenvalues in subsets labelled by $a$. They imply the equation $\xi_{a}(E)= \pm N g$. For finite $g$ and large $N$, given that the exponents grow linearly in $N$ with a coefficient that defines the Lyapunov exponent, the eigenvalues of the non-Hermitian matrix $\mathcal{H}(z)$ distribute along $M$ level lines

$$
\gamma_{a}(E)=g
$$

The distribution along arcs is shown in Fig. 2, for large $z$. For small $z$ the pattern of the eigenvalue distribution is complicate and intertwined.

For $g$ small enough, in continuity with the description given for the periodic case, the eigenvalues still belong to the real axis, outside their periodicity bands. By increasing $g$, eigenvalues with the same label $a$ pairwise approach until a pair 
condenses. Correspondingly, an extremum is reached for the function $\Delta_{a}(E)$ and the pair of eigenvalues acquires opposite imaginary parts. There is a critical value $g_{a}$ of $g$ for this to happen for each label $a$ :

$$
\Delta_{a}^{\prime}\left(E_{a}\right)=0 \quad \Delta_{a}\left(E_{a}\right)=2 \cosh \left(N g_{a}\right)
$$

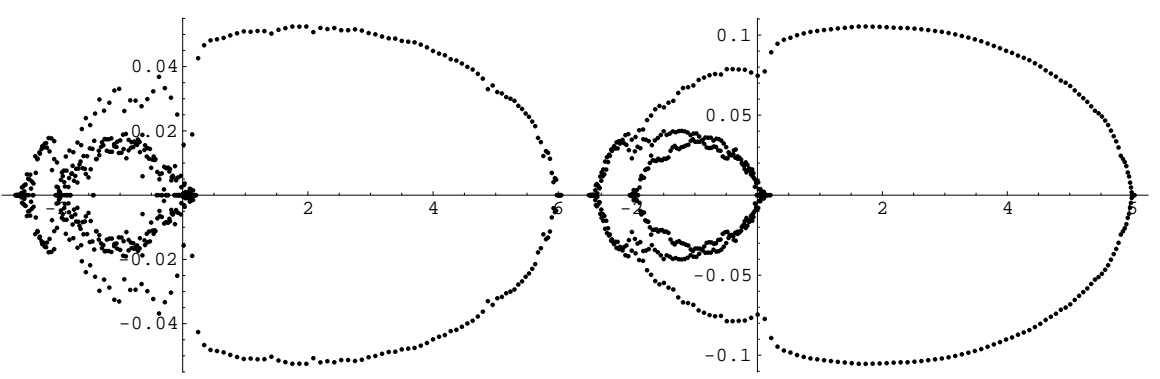

Fig.2 The 600 eigenvalues of an eptadiagonal matrix $(M=3, N=200)$, with diagonal uniform disorder $\left|a_{i}\right|<0.5$, unit off-diagonal elements, and $z=20$ (left), $z=390$ (right).

Finally, let us mention the case where $H_{i}=H_{i}^{\dagger}$ and $L_{i}$ are not real matrices. Another spectral identity is needed, which follows from spectral duality and the symplectic property [8]. It holds for any value of $z$ or $E$ in the complex plane:

$\operatorname{det}\left[T(E)+T(E)^{-1}-\left(z+\frac{1}{z}\right)\right]=\left|\operatorname{det} L_{1} \ldots L_{n}\right|^{-2} \operatorname{det}[E-\mathcal{H}(z)] \operatorname{det}\left[E-\mathcal{H}\left(\frac{1}{z}\right)\right]$

For $z=e^{i \varphi}$ the right side is zero in $2 N M$ real solutions, $E_{i}(\varphi)$ and $E_{i}(-\varphi)$, $i=1 \ldots N M$, that span the same $N M$ bands as $\varphi$ varies in $-\pi, \pi$. They are degenerate in $\varphi=0(y=2)$ and $\varphi=\pi(y=-2)$.

The strip $-2 \leq y \leq 2$ is thus crossed by $2 N M$ branches $y=\Delta_{a}(E)$ which join pairwise at the boundaries $y= \pm 2$ of the strip. Each pair, when intersected with the line $y=2 \cos \varphi$, determines the same band which the eigenvalue $E(\varphi)$ covers with different speeds in the two directions.

For $z= \pm e^{N g}$ we have again condensation of pairs of eigenvalues, which no longer become complex conjugate pairs. For large $N$ the eigenvalues move into $M$ level curves $\gamma_{a}(E)=g$ (the case $g<0$ leads to a different set of curves since in this case Lyapunov exponents need not be opposite pairs).

\section{Conclusion}

The spectral duality is a simple identity that links the eigenvalues of a matrix with block-tridiagonal structure to those of the corresponding transfer matrix. I have deduced some analytic properties for the exponents (sum of exponents, $N_{+}=N_{-}$, counting function). They involve a phase average on eigenvalues of the block-matrix. 
The large $N$ stability of exponents allows to describe the distribution of complex eigenvalues of the block matrix along arcs. Discriminants classify real eigenvalues (periodic case) in bands, and govern their motion and collisions.

These exact properties are hoped to allow a more analytic approach to the difficult study of Lyapunov spectra of transfer matrices, that are derived from an ensemble of random Hamiltonians. They also extend to block matrices some results which were known for purely tridiagonal matrices.

\section{References}

[1] G.Casati, I.Guarneri, F.M.Izrailev, L.Molinari and K.Zyczkowski: Periodic band random matrices, curvature and conductance in disordered media, Phys. Rev. Lett. 72 (1994) 2697.

[2] Y.V.Fyodorov and A.D.Mirlin: Scaling properties of localization in random band matrices: a $\sigma$-model approach, Phys. Rev. Lett. 67 (1991) 2405.

[3] B.Kramer and A.MacKinnon: Localization: theory and experiment, Rep. Prog. Phys. 56 (1993) 1469.

[4] S.Iida, H.A.Weidenmüller and J.Zuk: Statistical scattering theory, the supersymmetric method and universal conductance fluctuations, Ann. Phys. (N.Y.) 200 (1990) 219.

[5] E.Brézin, S.Hikami, A.Zee: Oscillating density of states near zero energy for matrices made of blocks with possible application to the random flux problem, Nucl. Phys. B 464 [FS] (1996) 411.

[6] T.Guhr, A.Müller-Groeling and H.Weidenmüller: Random Matrix Theory in Quantum Physics: Common Concepts, Phys. Rep. 299 (1998) 189.

[7] C.W.Beenakker: Random-matrix theory of quantum transport, Rev. Mod. Phys. 69 (1997) 731.

[8] L.Molinari: Transfer matrices and tridiagonal-block Hamiltonians with periodic and scattering boundary conditions, J. Phys. A: Math Gen. 30 (1997) 983.

[9] L.Molinari: Transfer matrices, non-hermitian Hamiltonians and resolvents: some spectral identities, J. Phys. A: Math. Gen 31 (1998) 8553.

[10] T.Kostyrko, M.Bartkowiak and G.D.Mahan: Reflection by defects in a tight-binding model of nanotubes, Phys. Rev B 59 (1999) 3241.

[11] T.Kostyrko: Transfer-matrix approach for modulated structures with defects, Phys. Rev. B 62 (2000) 2458. 
[12] T.Kostyrko: An analytic approach to the conductance and I-V characteristics of polymeric chains, J.Phys.: Condens. Matter 14 (2002) 4393.

[13] M.Hjort and S.Stafström: Localization in quasi-one-dimensional systems, Phys. Rev. B 62 (2000) 5245.

[14] N.Hatano and D.R.Nelson: Localization transition in quantum mechanics, Phys. Rev. Lett. 77 (1996) 570.

[15] N.M.Schnerb and D.R.Nelson: Winding numbers, complex currents and non-Hermitian localization, Phys. Rev. Lett. 80 (1998) 5172.

[16] J.Feinberg and A.Zee: Spectral curves of non-Hermitean Hamiltonians, Nucl. Phys. B 552 (1999) [FS] 599.

[17] I.Goldsheid and B.Khoruzhenko: Distribution of eigenvalues in nonHermitian Anderson models, Phys. Rev. Lett. 80 (1998) 2897.

[18] C.Mudry, P.W.Brouwer, B.I.Halperin, V.Gurarie and A.Zee: Density of states in the non-hermitian Lloyd model, Phys. Rev. B 58 (1998) 13539.

[19] I.Y.Goldsheid and B.A.Khoruzhenko: Regular Spacings of Complex Eigenvalues in the One-dimensional non-Hermitian Anderson model, math$\mathrm{ph} / 0209005$.

[20] A.Crisanti, G.Paladin and A.Vulpiani, Products of Random Matrices in Statistical Physics, Springer Series in Solid State Science 104 (1993).

[21] D.J.Thouless: A relation between the density of states and range of localization for one dimensional random systems, J. Phys. C: Solid State Phys. 5 (1972) 77.

[22] B.Derrida, J.L.Jacobsen and R.Zeitak: Lyapunov exponent and density of states of a non-Hermitian Schrödinger equation, J. Stat. Phys. 98 (2000) 31 .

[23] P.Markos: Universal scaling of Lyapunov exponents, J. Phys. A: Math. Gen. 30 (1997) 3441.

[24] T.Kottos, A.Politi, F.M.Izrailev and S.Ruffo: Scaling properties of Lyapunov spectra for the band random matrix model, Phys. Rev. E 53 (1996) $\mathrm{R} 5553$.

[25] I.S.Gradshteyn and I.M.Ryzhik, Table of Integrals, Series and Products, VI edition, Academic Press, (2000).

[26] K.Zyczkowski, L.Molinari and F.Izrailev: Level curvature and metalinsulator transition in 3d Anderson model, J. Phys. I France 4 (1994) 1469. 
[27] I.Guarneri, K.Zyczkowski, J.Zakrzewski, L.Molinari and G.Casati: Parametric spectral correlations of disordered systems in the Fourier domain, Phys. Rev. E 52 (1995) 2220.

[28] E.Korotyaev and I.V.Krasovsky: Spectral estimates for periodic Jacobi matrices, arXiv:math.SP/0205319v2. 